\title{
Rheological properties and fractal-rheology analysis of peanut protein isolate suspension
}

\author{
Chonghao $\mathrm{Bi}^{1}$, Shangyi Chi ${ }^{1}$, Zhe Hua², Dong $\mathrm{Li}^{3}$, Zhigang Huang ${ }^{1,4^{*}}$, Yi $\mathrm{Liu}^{1 *}$ \\ (1. School of Materials Science and Mechanical Engineering, Beijing Technology and Business University, Beijing 100048, China; \\ 2. School of Mechanical and Electronic Control Engineering, Beijing Jiaotong University, Beijing 100044, China; \\ 3. College of Engineering, China Agricultural University, Beijing 100083, China; \\ 4. Beijing Key Laboratory of Quality Evaluation Technology for Hygiene and Safety of Plastics, Beijing 100048, China)
}

\begin{abstract}
This study focuses on the non-linear rheological property and microstructure of peanut protein isolate (PPI) aggregation suspension. The impact of higher harmonics $\left(I_{3}\right.$ and $\left.I_{5}\right)$ on fundamental stress wave during large amplitude oscillatory shear test was studied. Rheological test show that storage modulus $G^{\prime}$ and loss modulus $G^{\prime \prime}$ increased with increasing PPI concentration. The non-linear viscoelastic properties of PPI suspension with different concentration were investigated. Using confocal laser-scanning microscopy method, this research explored the microstructure of PPI suspension as well as the fractal dimensions. The new critical strain indirect method combined with Wu-Morbidelli model to calculate the fractal dimension (2.9225) is very close to the actual fractal dimension (2.9206). Fourier Transform Rheology was adopted to get the new critical strain for fractal dimension calculation, which was proved to be feasible.
\end{abstract}

Keywords: peanut protein isolate suspension, rheological property, microstructure, fractal analysis

DOI: $10.25165 /$ j.ijabe. 20201306.5717

Citation: Bi C H, Chi S Y, Hua Z, Li D, Huang Z G, Liu Y. Rheological properties and fractal-rheology analysis of peanut protein isolate suspension. Int J Agric \& Biol Eng, 2020; 13(6): 220-226.

\section{Introduction}

The production of peanut in China is under a sustained development, and has ranked first in the world since the early $21 \mathrm{st}$ Century ${ }^{[1]}$. Peanut have become an important source of human protein intake due to the abundant protein and other nutrients. Peanut protein, which is now widely used in the food industry, is a major byproduct from peanut oil extraction industry ${ }^{[2]}$.

The protein content of peanuts is $25 \%-36 \%$, and the ratio of water-soluble and salt-soluble protein is approximately $1: 9^{[3]}$. Peanut protein is a kind of protein with homogeneous component and strong functional property, and its solubility, foaming property, water holding capacity, gel and other functional properties are very important in food processing ${ }^{[3]}$. Because of its high content of essential amino acid and unique flavor, peanut protein is considered as a good source of plant protein nutrition and has been getting more and more attention in the food industry ${ }^{[4]}$. The peanut protein isolate (PPI) can be extracted from peanut meal as an emulsifying agent or emulsion stabilizer in formulated foods ranging from sausages to vegetable creams ${ }^{[4-7]}$. PPI is favored by food manufacturer because of the active surface properties of its major constitutive proteins conarachin and arachin, which make up nearly $75 \%$ of the total protein ${ }^{[6]}$. The emulsifying properties of

\section{Received date: 2019-02-05 Accepted date: 2020-09-25}

Biographies: Chonghao Bi, PhD, Associated Professor, research interests: material property characteristics, Email: bichonghao@btbu.edu.cn; Shangyi Chi, Master, research interests: food machinery, Email: 920312701@qq.com; Zhe Hua, Master, research interests: test and control engineering, Email: 17121266@bjtu.edu.cn; Dong Li, PhD, Professor, research interests: agricultural material characterization, Email: dongli@cau.edu.cn.

*Corresponding author: Zhigang Huang, Professor, research interests: functional properties of food ingredients and food manufacturing machinery. Gengyun Building No.11, Fucheng Road, Haidian District, Beijing 100048, China. Tel: +86-10-68985249, Email: huangzg@btbu.edu.cn; Yi Liu, PhD, Associated Professor, research interests: characterization of agricultural material functional properties, Email: 1_y_61@ hotmail.com. peanut protein isolate have been studied and reported in detail. However, most of these studies have focused on quantifying and explaining the effects of different physical methods such as ultrasound and high pressure, or biological modifications (hydrolysis or transglutaminase crosslinking) on the physicochemical characteristics of peanut protein ${ }^{[8-11]}$.

In physiological processes such as chewing and swallowing, the intensity and speed of the stress applied to the material are quite strong and rapid. Similarly, the industrial production of food material will experience many short-term stress processes, such as mixing, pumping, liquid delivery. The large amplitude oscillatory shear test and Fourier transform analysis is considered to be the most important to the quantification of non-linear rheological parameters.

The large amplitude oscillatory shear test can be used to obtain and analyze the unconventional rheological parameters, such as the analysis of nonsinusoidal stress curve, frequency spectrum analysis. A variety of oscillatory flow phenomena in food production, processing, and transportation can be simulated and explained by large amplitude oscillatory shear tests which are closely related to the characteristics of the material being tested and very sensitive to the interaction of stress, shear, material structure and material composition $^{[12,13]}$. Fourier transform analysis can be used to analyze the nonsinusoidal stress curves obtained from the large amplitude shear test. This technique can be used to extract high order harmonics in the stress response curve and convert the time domain signal into a frequency domain signal to accomplish the quantification and analysis of the non-linear rheological data ${ }^{[12,14]}$.

Fractal theory has been applied in many fields, including natural science, social science, philosophy. The main method of fractal theory applied in the field of food material characterization is to calculate the fractal dimension which is usually used to describe the chaos and complexity of a gel or hydrocolloid system. There are two categories of methods for calculating fractal dimension: one is direct methods, including confocal laser scanning 
microscopy (CLSM), small angle $\mathrm{X}$ light scattering (SAOS) and dynamic light scattering (DLS) etc.; the other is indirect methods, which is to calculate fractal dimension based on the characteristic parameters obtained from rheology or acoustics test etc. ${ }^{[15-18]}$

The fractal-rheology is one of the most mature and common indirect methods to obtain the fractal dimension of samples that is not readily to measure by direct methods. Scientists have proposed a number of theoretical models for protein and other polymers $^{[19-22]}$. Among them, the model of Shih is widely used to calculate the fractal dimension by the critical strain in the rheological test, and the hydrocolloid is divided into two types: the strong gel and the weak gel ${ }^{[21]}$. Furthermore, $\mathrm{Wu}$ and Morbidelli ${ }^{[23]}$ optimized the model of Shih, and introduced new parameters, then the hydrocolloid can be divided into three kinds: strong gel, transition gel, weak gel.

The models proposed by previous scholars extend the application range of fractal-rheology, however, there are still problems that need to be improved in terms of accuracy and applicability. In this manuscript, we work on the characterization of non-linear rheological property of PPI suspension and focus on finding a more applicable fractal-rheology model for PPI suspension.

\section{Materials and methods}

\subsection{Materials and equipment}

Peanut protein isolate (S31067, BR grade) was obtained from Shanghai Yuanye Bio-Technology Co., Ltd. (Shanghai, China); Rhodamine B (BS) was purchased from Beijing Yinghai Fine Chemical Industry (Beijing, China).

Rheometer (AR2000 ex, TA Instruments Company, USA), confocal scanning microscope (LEICA TCS SP5 II, Germany LEICA instruments, Germany), magnetic stirrer (85-2, Jiangsu Jintan Jingda Instrument, China), and analytical balance (AL 204, Mettler-Toledo (Switzerland) were used for sample tests.

\subsection{Sample preparation}

A total of $27 \mathrm{~g}$ PPI powder was dissolved in $100 \mathrm{~mL}$ deionized water ( $\mathrm{pH} 6.4-7.0)$ at $20^{\circ} \mathrm{C}$ by stirring for $2 \mathrm{~h}$ using a magnetic stirrer $(500 \mathrm{r} / \mathrm{min})$. The peanut protein isolate aggregation suspension was diluted to concentrations of $23 \%, 24 \%, 25 \%, 26 \%$ and $27 \%(\mathrm{v} / \mathrm{w})$. The suspensions at different concentrations were stored at $4{ }^{\circ} \mathrm{C}$ overnight for complete hydration. Before testing, the overnight samples needed to be stirred for $5 \mathrm{~min}$ on a magnetic stirrer and warmed to $20^{\circ} \mathrm{C}$ to ensure the homogeneity of the samples.

For the CLSM test, the PPI suspension sample was stained with an aqueous solution of Rhodamine B $(0.05 \mathrm{~mL}$ of $0.01 \%(\mathrm{w} / \mathrm{w})$ Rhodamine $\mathrm{B}+5 \mathrm{~mL}$ stock solution of peanut protein isolate suspension). The stained stock solution was added onto a concave slide and sealed. Samples were kept in dry and dark place. The excitation wavelength of Rhodamine B is $543 \mathrm{~nm}$, and the emission wavelength of light is between $561 \mathrm{~nm}$ and $680 \mathrm{~nm}$.

\subsection{Rheological tests}

\subsubsection{Large Amplitude Oscillatory Shear (LAOS) tests}

LAOS test was operated at strain ranging from $0.01 \%$ to $1000 \%$ at a fixed angular frequency of $6.28 \mathrm{rad} / \mathrm{s}$. The temperature is controlled at $20^{\circ} \mathrm{C}$, and the strain and stress original data points were recorded at a rate of 10 points per decade.

When the strain is small (typically $<1 \%$ ), the stress response waveform is sinusoidal, and the influence of higher harmonic to fundamental wave is not obvious. When the strain amplitude increases beyond the linear viscoelastic regime (typically $>1 \%$ ), the stress waveform becomes distorted and the signal becomes non-sinusoidal, which means that the effect of higher harmonic begins to affect the fundamental wave ${ }^{[24]}$. Fourier transform analysis is the relatively mature methods to acquire the entire frequency spectra covering the non-linear stress response $\mathrm{e}^{[22]}$. The data of odd harmonics (i.e., $I_{1}, I_{3}, I_{5} \ldots$, frequency of harmonics at odd times of fundamental wave) obtained from LAOS and applied in Fourier transform analysis were used to explain the non-linear behavior of PPI suspensions.

The time domain nonsinusoidal stress response was transferred into frequency domain spectra using the Fourier transformation and the stress was decomposed into a series of higher harmonics as given by Equation (1):

$$
\sigma=A \sin \left(\omega_{1} t+\delta_{1}\right)+B \sin \left(\omega_{2} t+\delta_{2}\right)+C \sin \left(\omega_{3} t+\delta_{3}\right)+\ldots
$$

where, $\sigma$ is the nonsinusoidal stress wave, $\mathrm{Pa} ; \omega_{1,2,3} \ldots$ and $\delta_{1,2,3} \ldots$ are the angular frequency $(\mathrm{rad} / \mathrm{s})$ and phase angle $\left(^{\circ}\right)$ of each harmonic, respectively; A, B, C are constants.

Moreover, when the strain is in the large amplitude regime, the stress waveform not only becomes nonsinusoidal but also goes out of phase. For simplicity, this research studied the odd harmonics $\left(I_{3}, I_{5}\right)$ from Fourier transformation instead of covering the entire complex higher harmonics spectra, because $I_{3}$ and $I_{5}$ had the greatest and nonignorable contribution to the fundamental wave.

Lissajous curve is a close loop that can be used to analyze the ratio of elasticity/viscosity, viscous loss and the trend of shear rate. Two limiting cases of the Lissajous curve are the Hooke elastic solid and the Newton fluid. For Hooke elastic solid, the Lissajous curve is a straight line with a slope of $G$; for Newton fluid, the Lissajous curve is an elliptic curve with respect to an axis of symmetry. The ratio of the viscosity and elasticity of the material is determined by the area of the Lissajous curve. The line inside the Lissajous curve indicates the effect of shear rate on the stress response.

\subsubsection{CLSM test and fractal analysis}

The confocal laser scanning microscope comprises an inverted microscope (Leica DMI6000) and He-Ne/visible light laser source (Leica Microsystems CMS GmbH Germany). An objective lens of $20 \times 0.85$ (NA) was used to observe the microstructure of the PPI suspensions.

Direct method to obtain fractal dimension: the tricolour (RGB) images were transformed to 8-bit grey images with a scale of $1024 \times 1024$ pixels. A black-white binarization was performed using the mid-value of the grey level histogram of each image as a threshold ${ }^{[25]}$. A box-counting method was applied to calculate the fractal dimension $\left(D_{f}\right)$ of the microstructure of PPI suspension samples ${ }^{[26]}$. This method is based on the scale rule given by Equations (2) and (3):

$$
\begin{gathered}
D=-\log N_{\varepsilon} / \log \varepsilon \\
D_{f}=D_{p}+1
\end{gathered}
$$

At a given scale $\varepsilon, N_{\varepsilon}$ is the number of boxes that contains the PPI suspensions' network image. $D_{p}$ and $D_{f}$ are the values of fractal dimensions in two and three dimensions, respectively. $D_{p}$ lies between 1 and 2 as it is calculated from a two-dimensional space. To characterize the three-dimension structure, an extra dimension is required ${ }^{[27]}$.

Rheology-fractal model: many scientists have developed the rheology-fractal models applying to gels, polymers, and hydrocolloids. Shih model ${ }^{[21]}$ and Wu-Morbidelli model ${ }^{[23]}$ were selected for PPI microstructure characterization.

In Shih model: the storage modulus and the critical strain are proportional to the volume fraction of the particles. Based on the 
force in the inner chain and among the chains of the protein molecule, the gel was divided into two types: strong-link regime and weak-link regime.

For a strong-link regime:

$$
\begin{aligned}
& G_{i}^{\prime} \propto \varphi^{(D+x) /\left(D-D_{f}\right)} \\
& \gamma_{C} \propto \varphi^{-(1+x) /\left(D-D_{f}\right)}
\end{aligned}
$$

For a weak-link regime:

$$
\begin{gathered}
G_{i}^{\prime} \propto \varphi^{(D-2) /\left(D-D_{f}\right)} \\
\gamma_{C} \propto \varphi^{1 /\left(D-D_{f}\right)}
\end{gathered}
$$

where, $G_{i}{ }^{\prime}, \gamma_{c}$ are the storage modulus and strain value at the critical point,respectively. $\varphi$ is the volume fraction of the main gel component. $D, D_{f}$ and $x\left(1<x<D_{f}\right)$ are the Euclidean dimension, fractal dimension, gel skeleton dimension, respectively ${ }^{[21]}$.

In Wu-Morbidelli model: the fractal model of $\mathrm{Wu}$ and Morbidelli optimized the fractal model of Shih, introducing two new parameters: one is the intermediate variable $\beta$, the other is a constant $\alpha(0<\alpha<1)$. When $\alpha=0$, the gel network structure is strong, which indicates strong gel. When $\alpha=1$, the gel network structure is weak, which indicates weak gel. When $0<\alpha<1$, the gel network structure is in the transition region, which indicates a transition gel ${ }^{[23]}$.

$$
\begin{gathered}
G_{i}^{\prime} \propto \varphi^{\beta /\left(D-D_{f}\right)} \\
\gamma_{C} \propto \varphi^{(D-\beta-1) /\left(D-D_{f}\right)} \\
\beta=(D-2)+(2+x)(1-\alpha)
\end{gathered}
$$

where, $\beta$ is an intermediate variable. $D, D_{f}$ and $x\left(1<x<D_{f}\right)$ are the Euclidean dimension, fractal dimension, gel skeleton dimension, respectively. When calculating the fractal dimension, the two empirical estimates of $x=1$ and $x=1.3$ are usually used to calculate the parameter $\alpha^{[23]}$.

\subsection{Data processing and statistical analysis}

The box-counting method was conducted by the public domain software ImageJ 1.44p (National Institutes of Health, USA). Each experiment was carried out at least in triplicate, and the results were presented in terms of mean \pm standard deviation. The SPSS statistical package (SPSS 16.0, IBM Corporation, USA) was used to determine the significant difference between two mean values at the $95 \%$ confidence level $(p<0.05)$.

\section{Results and discussion}

\subsection{Results from LAOS tests}

Figure 1a depicts the effect of the strain on the storage modulus $\left(G^{\prime}\right)$ of PPI suspension at different concentration. As shown in Figure 1a, the variation of the storage modulus $G^{\prime}$ with different PPI concentration has a similar trend. When the strain was less than $0.3 \%$, the values of the storage modulus $G^{\prime}$ of PPI suspensions at different concentrations did not change with the strain increasing. With the increase of the strain, which was greater than $0.3 \%$, the storage modulus $G^{\prime}$ began to decline. The suspension with higher PPI concentration contains more solute, and the formation of the suspension structure is more stable and more elastic. Additionally, it can be seen that the gradient of PPI concentration was $1 \%$, however, the difference between the $G^{\prime}$ values of these suspension samples was very large. For example, the $G^{\prime}$ value of the linear viscoelastic region of $24 \%$ PPI suspension was $60 \mathrm{~Pa}$, while that of $25 \%$ PPI was $100 \mathrm{~Pa}$. The strain value at the turning point of the transition from the linear viscoelastic region to the non-linear region is defined as the critical strain $\left(G_{\text {critical }}^{\prime}=\right.$ $\left.95 \% G_{L V R}^{\prime}\right)$, which will be used to characterize the scaling behaviour of the suspension in Section 3.2.2.

Figure $1 \mathrm{~b}$ presents the effect of the strain on the loss modulus $\left(G^{\prime \prime}\right)$ of PPI suspensions at different concentrations. When the strain was greater than $2 \%$, the loss modulus began to decrease. The $G^{\prime \prime}$ value of the linear viscoelastic region is generally concentrated in the range of 3-30 Pa. The $G^{\prime \prime}$ value of the linear viscoelastic region was lower than $G^{\prime}$ value of the linear viscoelastic region, which indicates that the elasticity of PPI suspension is dominant. Comparing the trends of $G^{\prime}$ and $G^{\prime \prime}$, PPI suspension performed the characteristics of polymer Type $I$ structure according to the structure model of Hyun ${ }^{[12]}$. This illustrates that it is easy for the suspension to collapse when the strain is large, and difficult for molecules to have a chance to rebuild their structures.

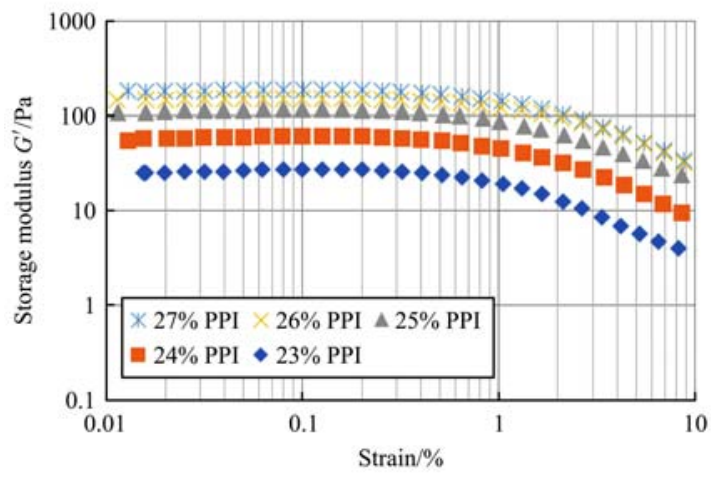

a. Storage modulus $\left(G^{\prime}\right)$

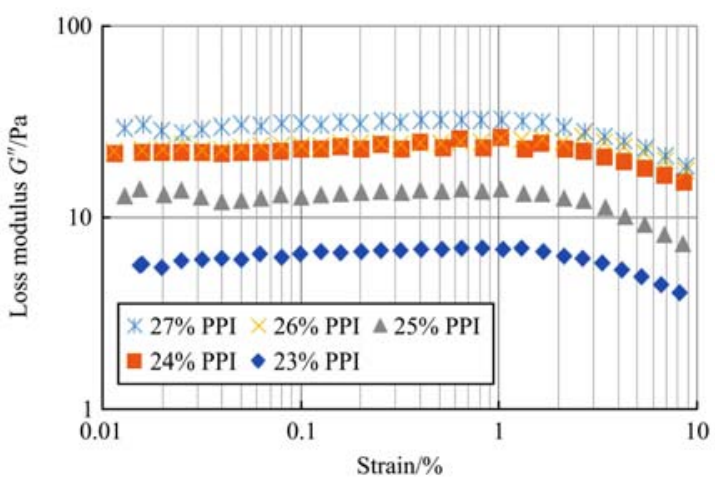

b. Loss modulus $\left(G^{\prime \prime}\right)$

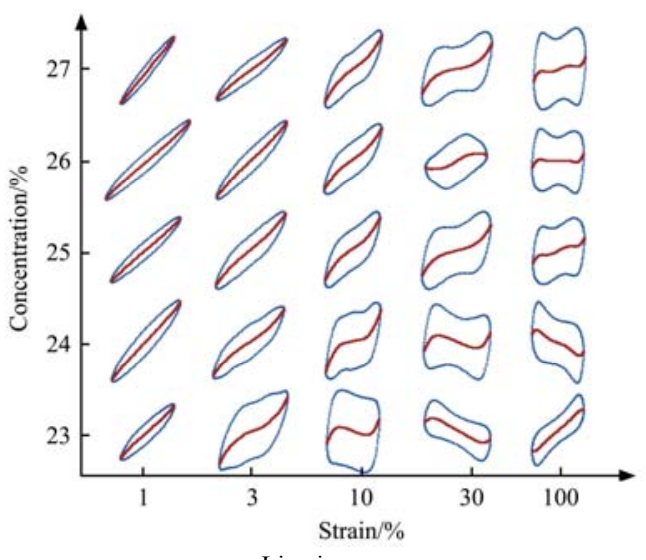

c. Lissajous curves

Figure 1 Effect of the strain on the storage modulus $\left(G^{\prime}\right)$, the loss modulus $\left(G^{\prime \prime}\right)$ and the Lissajous curves of peanut protein isolate suspension at different concentrations

Figure 1c shows the effect of the strain on the Lissajous curves of PPI suspensions at different concentrations. The Lissajous curves have been regularized. When the applied strain was $1 \%$, the area of the Lissajous curves was relatively small, which means 
that the elasticity/viscosity ratio was relatively high, and viscous loss was small. When the concentrations were constant, the area of the Lissajous curve increased with the increase of applied strain, which illustrated that the proportion of viscosity was increasing, as well as viscous loss. Furthermore, when the concentrations were constant, the line inside the Lissajous curve changed with the increase of strain. When line changed from a straight one to a curve, it meant that the higher harmonic began to significantly affect the stress response. The phenomenon also indicates that the strain rate changing with applied stress varies with the PPI concentration increasing. It is noted that, for $23 \%$ PPI suspension, the area of the Lissajous curve reached a maximum value in a strain range of $3 \%-10 \%$; for $24 \%$ PPI suspension, the area of the Lissajous curve reached a maximum value in a strain range of $10 \%-30 \%$; for $25 \%, 26 \%$ and $27 \%$ PPI suspension, the the area of the Lissajous curve reached a maximum value in a strain range of $30 \%-100 \%$. Hence, the applied strain, at which the PPI suspension shows maximum viscosity property, tended to be smaller when the PPI concentration was lower.

Figure 2 depicts the Fourier transform of original strain-stress rheological data of $26 \%$ PPI sample. The value of $I_{3 / 1}$ had a rise at the beginning, then the $I_{3 / 1}$ reached a maximum value of 0.3175 at the strain of $64.76 \%$. When the strain reached $6.31 \%$, the effect of $I_{3 / 1}$ on the fundamental wave began to be nonignorable $\left(I_{3 / 1}>0.05\right)$. This phenomenon demonstrates that the suspension structure exhibits an irreversible structural change when the strain reaches $6.31 \%$. As the strain continued to increase, the value of $I_{3 / 1}$ decreased gradually. The maximum value of $I_{3 / 1}$ is exactly at the end point of the falling area of the loss modulus $G^{\prime \prime}$, which is not coincidental, as shown in previous study of soy protein isolate suspension ${ }^{[28]}$. In addition, the end of the falling area of $G$ " was caused by the influence of higher harmonics.

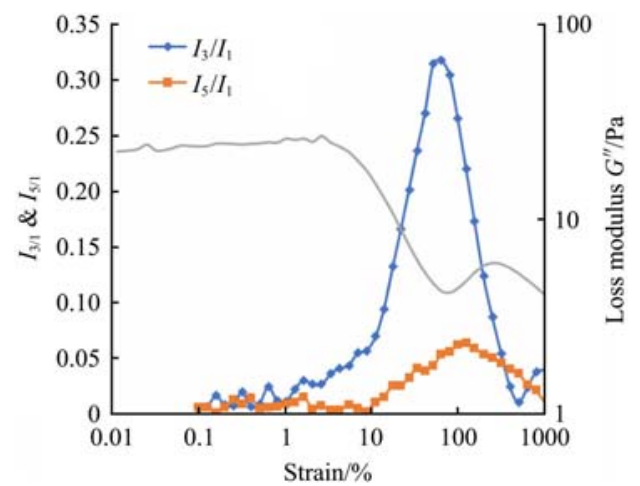

Figure 2 Effects of the strain on Fourier transform of peanut protein isolate suspension at $26 \%$ concentration

\subsection{Microstructure and fractal dimension of PPI suspensions}

\subsubsection{Microstructure of PPI suspensions}

The microstructure of PPI suspension at $25 \%$ concentration by confocal scanning microscopy was performed in Figure 3a. The scale of the image is 1:200. The green zone is a protein-rich area stained by Rhodamine B, and the black region is the pore between the protein networks. As seen from Figure 3a, the feature size of PPI is significantly larger than the pore size, which indicates that the extent of the formation of the protein aggregation in the PPI suspension is relatively strong.

Figure $3 \mathrm{~b}$ is the CLSM image of the PPI suspension treated by black-white binarization. The binary CLSM image can be used to calculate the fractal dimension of the PPI suspension directly by the box-counting method. In Figure $3 \mathrm{c}$, the box-counting

experimental data can be fitted very well with a regression curve. The fractal dimension of PPI suspension calculated by the direct method is 1.9206 .

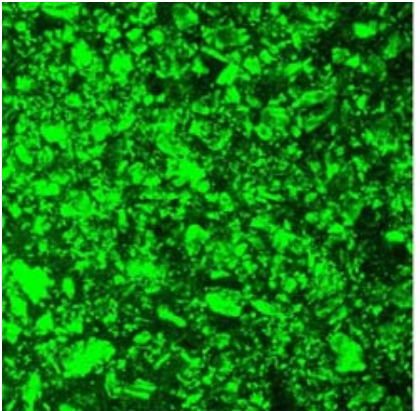

a. Microscopic images

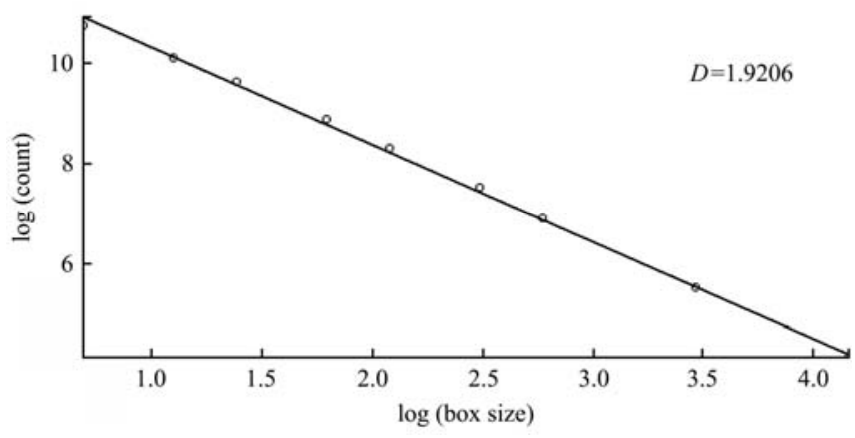

c. Fractal dimension

Figure 3 Microscopic images, CLSM image and fractal dimension of peanut protein isolate suspension at $25 \%$ concentration

\subsubsection{Fractal analysis of PPI suspensions}

With the increase of concentration, the storage modulus $\left(G^{\prime}\right)$ of the linear viscoelastic region increased gradually (Table 1$)$, which indicated that the strain resistance ability of PPI suspension increased with concentration increasing. The concentration and critical strain are positively correlated. Therefore, the exponent of the power function in Figure $4 \mathrm{~b}$ is positive.

Table 1 Storage modulus and critical strain of the linear viscoelastic region of peanut protein isolate suspensions

\begin{tabular}{ccc}
\hline Concentration $/ \%$ & Storage modulus $G^{\prime} / \mathrm{Pa}$ & Critical strain/\% \\
\hline 23 & $26.573 \pm 0.943$ & $0.415 \pm 0.010$ \\
24 & $60.154 \pm 1.965$ & $0.429 \pm 0.016$ \\
25 & $113.233 \pm 4.693$ & $0.450 \pm 0.023$ \\
26 & $149.982 \pm 3.412$ & $0.453 \pm 0.027$ \\
\hline
\end{tabular}

Figures $4 \mathrm{a}$ and $4 \mathrm{~b}$ present the scaling behavior of storage modulus $G^{\prime}$ in linear viscoelastic region and critical strain $\gamma_{c}$ obtained from LAOS test. The $G^{\prime}$ and $\gamma_{c}$ of PPI suspensions at different concentrations showed a greatly good power law function:

$$
\begin{aligned}
G^{\prime} & \propto C^{n} \\
\gamma_{c} & \propto C^{m}
\end{aligned}
$$

where, $n$ is a power law index of $G^{\prime}$; and $m$ is a power law index of $\gamma_{c}$. As seen from Figures $4 \mathrm{a}$ and $4 \mathrm{~b}, n$ is 14.302 ; and $m$ is 0.7562 for PPI suspension. As $m$ value is positive, it can be seen from the Shih model that the structure connection of PPI suspension belongs to a weak-link regime.

As shown in Figures 4a and $4 \mathrm{~b}$

$$
\begin{aligned}
& G_{i}^{\prime}=1 E-18 C^{14.302} \\
& \gamma_{C}=0.0389 C^{0.7562}
\end{aligned}
$$

Therefore, the process of calculating fractal dimension is as follows: 


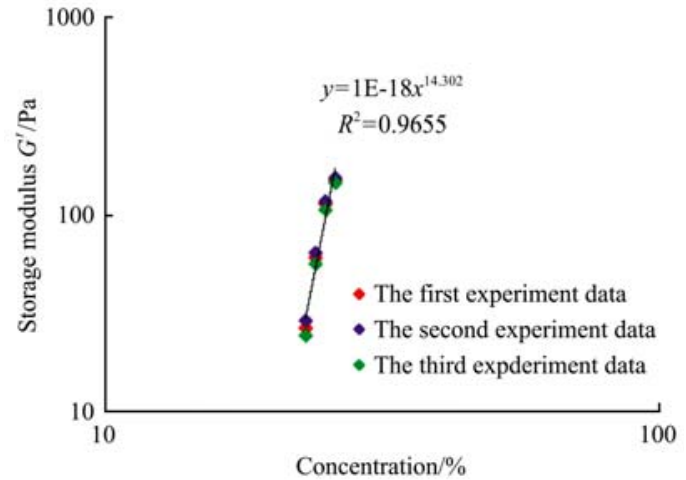

a. Storage modulus $G^{\prime}$
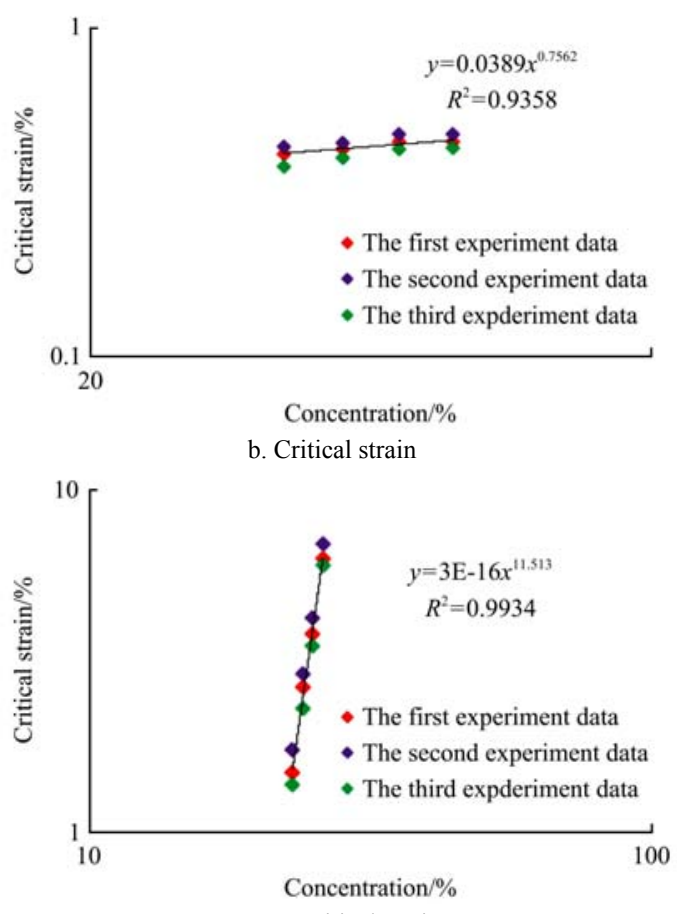

c. New critical strain

Figure 4 Regression curve of storage modulus $G^{\prime}$, critical strain and new critical strain of PPI suspensions at different concentrations

(1) Shih model:

As seen from Figure $4 \mathrm{~b}, m$ is 0.7562 . Therefore, the weak gel equation of the Shih model (Equations (6) and (7)) has been chosen.

Then, $\quad\left\{\begin{array}{l}\frac{D-2}{D-D_{\mathrm{f}}}=14.302 \\ \frac{1}{D-D_{f}}=0.7562\end{array}\right.$

Because $D=3$ (three-dimensional structure)

$$
\text { So, } \quad\left\{\begin{array}{l}
\frac{1}{D-D_{\mathrm{f}}}=14.302 \\
\frac{1}{D-D_{f}}=0.7562
\end{array}\right.
$$

Therefore, $\quad\left\{\begin{array}{l}D_{f}=2.93 \text { or } \\ D_{f}=1.6776\end{array}\right.$

(2) Wu-Morbidelli model:

According Equations (8)-(10),

$$
\left\{\begin{array}{l}
\frac{1+(2+x)(1-\alpha)}{3-D_{\mathrm{f}}}=14.302 \\
\frac{1-(2+x)(1-\alpha)}{3-D_{f}}=0.7562
\end{array}\right.
$$

If $x=1$, then

$\left\{\begin{array}{l}\frac{1+3(1-\alpha)}{3-D_{f}}=14.302 \\ \frac{1-3(1-\alpha)}{3-D_{f}}=0.7562\end{array}\right.$

Therefore,

$$
\left\{\begin{array}{l}
\alpha=0.7 \\
D_{f}=2.8672
\end{array}\right.
$$

If $x=1.3$, then

$$
\left\{\begin{array}{l}
\frac{1+3.3(1-a)}{3-D_{f}}=14.302 \\
\frac{1-3.3(1-a)}{3-D_{f}}=0.7562
\end{array}\right.
$$

Therefore,

$$
\left\{\begin{array}{l}
\alpha=0.7274 \\
D_{f}=2.8672
\end{array}\right.
$$

In summary, because $D_{f}=1.6776<2$ does not conform to the actual situation, the solution of Shih model is $D_{f}=2.93$. Furthermore, the solution of Wu-Morbidelli model is $D_{f}=2.8672$ which is consistent with the actual situation.

3.2.3\#\#Fractal model optimization

A critical strain represents the strain corresponding to the turning point that from the linear viscoelastic region to the non-linear viscoelastic region. To define a critical strain, Shih and Wu-Morbidelli models choose the strain of the data point at $G^{\prime}=0.95 \times G_{i}{ }^{\prime}$. However, when the harmonic generation $\left(I_{3 / 1}\right)$ begins to increase, the corresponding strain may be used as a new critical point to calculate the fractal dimension in rheology-fractal analysis. The following calculation is based on this conjecture.

Figure 5 presents the effect of strain on the third higher harmonic $\left(I_{3 / 1}\right)$ of PPI suspensions at different concentrations. When the applied strain was less than $1 \%$, the values of $I_{3 / 1}$ for PPI suspension at different concentrations were very small (less than 0.03 ), which meant that the influence of higher harmonic to fundamental stress wave was too small to be noted. When strain value rises to the range of $1 \%-10 \%$, the values of $I_{3 / 1}$ for PPI suspensions at different concentrations become to increase, which indicates that the influence of the higher harmonic in this strain section could not be ignored. Therefore, we chose the data points at $I_{3 / 1}=0.05$ to be the new critical points, and the corresponding strains were regarded as new critical strains.

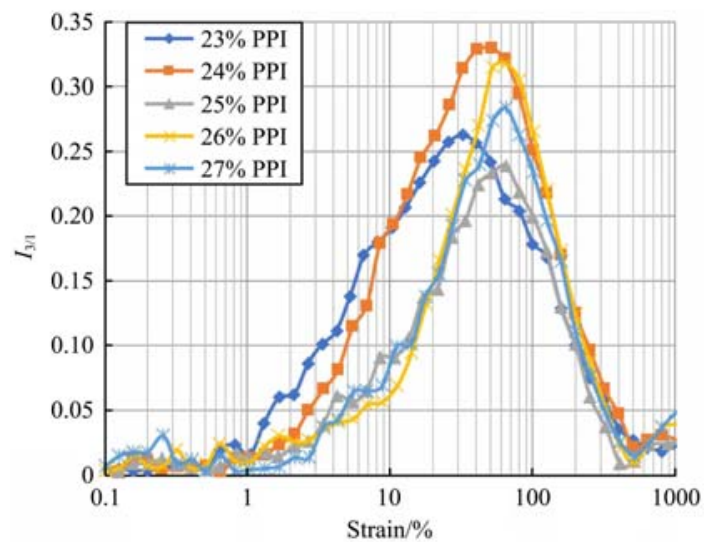

Figure 5 Effects of the strain on high order harmonic $\left(I_{3 / 1}\right)$ of peanut protein isolate suspensions at different concentrations

It can be seen from Table 2 that with the increase in protein concentration, the storage modulus of the linear viscoelastic region and new critical strain both increases gradually, which demonstrates a positive correlation between PPI concentration and new critical strain. Therefore, the exponent of the power function 
in Figure $4 \mathrm{c}$ is positive. It is noted that the critical strain values in Table 2 are larger than those in Table 1, which provides the possibility to calculate fractal dimensions more accurately.

Table 2 Critical strain from Fourier transform analysis and the storage modulus of PPI suspensions at different concentrations

\begin{tabular}{ccc}
\hline Concentration $/ \%$ & Storage modulus $G^{\prime} / \mathrm{Pa}$ & Critical strain/\% \\
\hline 23 & $26.573 \pm 0.943$ & $1.484 \pm 0.013$ \\
24 & $60.154 \pm 1.965$ & $2.661 \pm 0.021$ \\
25 & $113.233 \pm 4.693$ & $3.807 \pm 0.032$ \\
26 & $149.982 \pm 3.412$ & $6.314 \pm 0.036$ \\
\hline
\end{tabular}

Using the new critical strain, the scaling behaviour of storage modulus $G^{\prime}$ and the critical strain also showed a very good power law function with the concentration of PPI:

As shown in Figures $4 \mathrm{a}$ and $4 \mathrm{c}$

$$
\begin{aligned}
& G_{i}^{\prime}=1 E-18 C^{14.302} \\
& \gamma_{C}=3 E-16 C^{11.513}
\end{aligned}
$$

Therefore, the calculation process is still the same, the only change is from 0.7562 to 11.513 in Equations (15) and (16).

Table 3 shows that the Shih model is more suitable than the Wu-Morbidelli model to estimate the fractal dimension of PPI suspensions before optimizing the rheological fractal model. After the new critical strain is introduced to calculate the fractal dimension, the values of the fractal dimensions calculated by these two models are all closer to the values obtained from the microscopic images. Wu-Morbidelli model along with new critical strain makes the indirect method result (2.9225) very close to the actual fractal dimension value (2.9206), the error value is 0.0019 . The conjecture of this study is proved to be successful, and the new critical strain obtained from FT rheology method can improve the accuracy of the indirect fractal dimension calculation method. It is also demonstrated that Wu-Morbidelli model combined with new critical strain is more suitable to characterize the fractal properties of PPI suspension.

Table 3 Comparison of fractal dimensions obtained from microscopic images and rheological methods

\begin{tabular}{lccc}
\hline \multicolumn{1}{c}{ Rheological fractal model } & Non-optimized $D_{f}$ & Optimized $D_{f}$ & Actual $D_{f}$ \\
\hline Shih model & 2.9300 & 2.9300 or 2.9130 & \\
Wu-Morbidelli model $(x=1)$ & 2.8672 & 2.9225 & 2.9206 \\
Wu-Morbidelli model $(x=1.3)$ & 2.8672 & 2.9225 & \\
\hline
\end{tabular}

\section{Conclusions}

In the LAOS test, PPI suspension conformd the characteristics of polymer Type I structure, which meant it was easy for the suspension to collapse when the strain was large, and it was difficult for molecules to have a chance to rebuild their structures. From Lissajous curve analysis, the strain rate changing with applied stress varied with the PPI concentration increasing, and the applied strain, at which the PPI suspension shows maximum viscosity property, tended to be small when the PPI concentration was lower. In higher harmonics curve, it is noted that the maximum value of $I_{3 / 1}$ is exactly at the end point of the falling area of the loss modulus $G^{\prime \prime}$. As to the microstructure of PPI suspension, the images from CLSM indicated that the extent of the formation of the protein aggregation in the PPI suspension was relatively strong. The fractal dimension of PPI suspension calculated from the CLSM images was 2.9206. Using a new critical strain obtained from FT rheology, the accuracy of scaling behavior was improved. The experimental result demonstrated that Wu-Morbidelli model combined with new critical strain was more suitable to characterize the fractal properties of PPI suspension.

\section{Acknowledgements}

This research project was supported by General Project of Scientific Research Program of Beijing Education Commission (KM201910011003, KM201910011001), Joint Program of Beijing Natural Science Foundation Committee and Beijing Education Committee (KZ201810011017), and Beijing Excellent Talent Training Project (2017000020124G100).

\section{[References]}

[1] Li M Z, Yao K, Jia D Y, He Q, Lai B L. Functional components and comprehensive utilization of peanut. Chinese oil, 2004; 29(9): 13-15.

[2] Cao K G. Comprehensive utilization of peanut resources. Jiangxi Food Industry, 2002; 1: 15-17.

[3] Liu D C, Zhang W N, Hu X H. Study on preparation and functional properties of peanut protein. Journal of Wuhan Polytechnic University, 2001; 4: 1-4.

[4] Ghatak S K, Sen K. Peanut proteins: applications, ailments and possible remediation. Journal of Industrial and Engineering Chemistry, 2013; 19(2): 369-374.

[5] Zhao X Y, Chen J, Du F L. Potential use of peanut byproducts in food processing: a review. Food Sci. Technol, 2012; 49: 521-529.

[6] Neucere N J, Conkerton E J. Some physicochemical properties of peanut protein isolates. Agric. Food Chem, 1978; 26: 683-686.

[7] Dong X H, Zhao M M, Shi J, Yang Bao, Li J, Luo D H, et al. Effects of combined high pressure homogenization and enzymatic treatment on extraction yield, hydrolysis and function properties of peanut proteins. Innovative Food Science and Emerging Technologies, 2011; 12(4): 478-483.

[8] Fekria A M, Isam A M A, Suha O A, Elfadil E B. Nutritional and functional characterization of defatted seed cake flour of two sudanese groundnut (Arachis hypogaea) cultivars. Int. Food Res, 2012; 19: 629-637.

[9] Hu X, Zhao M M, Li L H, Yang B, Yang X Q, Wang H Y, et al. Emulsifying properties of cross-linking between proteins extracted from cold/hot pressed peanut meal and hydrolysed fish (Decapterus maruadsi) proteins. Int. J. Food Prop, 2014; 17: 1750-1762.

[10] He X H, Liu H Z, Liu L, Hu H, Wang Q. Effects of high pressure on the physicochemical and functional properties of peanut protein isolates. Food Hydrocoll, 2014; 36: 123-129.

[11] Li C, Huang X J, Peng Q, Shan Y Y, Xue F. Physicochemical properties of peanut protein isolate-glucomannan conjugates prepared by ultrasonic treatment. Ultrason Sonochem, 2014; 21: 1722-1727.

[12] Hyun K, Kim S H, Ahn K H, Lee S J. Large amplitude oscillatory shear as a way to classify the complex fluids. Journal of Non-Newtonian Fluid Mechanics, 2002; 107(1-3): 51-65.

[13] Leblanc J L. Non-linear viscoelastic characterization of molten thermoplastic vulcanizates (TPV) through large amplitude harmonic experiments. Rheologica Acta, 2007; 46(80): 1013-1027.

[14] Sun W X, Yang Y R, Wang T, Liu X X, Wang C Y, Tong Z. Large amplitude oscillatory shear rheology for non-linear viscoelasticity in hectorite suspensions containing poly (ethylene glycol). Polymer, 2011; 52(6): 1402-1409.

[15] Hagiwara T, Kumagai H, Nakamura K. Fractal analysis of aggregates formed by heating dilute BSA solutions using light scattering methods. Bioscience, Biotechnology and Biochemistry, 1996; 60(11): 1757-1763.

[16] Hagiwara T, Kumagai H, Matsunaga T, Nakamura K. Analysis of aggregate structure in food protein gels with the concept of fractal. Bioscience, Biotechnology, and Biochemistry, 1997; 61(10): 1663-1667.

[17] Matsumoto T, Kawai M, Masuda T. Viscoelastic and SAXS investigation of fractal structure near the gel point in alginate aqueous systems. Macromolecules, 1992; 25(20): 5430-5433.

[18] Wu H, Xie J J, Lattuada M, Morbidelli M. Scattering structure factor of colloidal gels characterized by static light scattering, small-angle light scattering, and small-angle neutron scattering measurements. Langmuir, 2005; 21(8): 3291-3295. 
[19] Bremer L G B, Bijsterbosch B H, Schrijvers R, Vliet V T, Walstra P. On the Fractal Nature of the Structure of Acid Casein Gels. Colloids and Surfaces, 1990; 51: 159-170

[20] Mellema M, Opheusden V J H J, Vliet V T. Categorization of rheological scaling models for particle gels applied to casein gels. Journal of Rheology, 2002; 46(1): 11-29.

[21] Shih W H, Shih W Y, Kim S I, Liu J, Aksay I A. Scaling behavior of the elastic properties of colloidal gels. Physical Review A, 1990; 42(8): 4772-4779.

[22] Wilhelm M, Maring D, Spiess H W. Fourier-transform rheology. Rheologica Acta, 1998; 37(4): 399-405.

[23] Wu H, Morbidelli M. A model relating structure of colloidal gels to their elastic properties. Langmuir, 2001; 17(4): 1030-1036.
[24] Le Grand A, Petekidis G. Effects of particle softness on the rheology and yielding of colloidal glasses. Rheologica Acta, 2008; 47(5-6): 579-590.

[25] Bi C H, Li D, Wang L J, Gao F, Adhikari B. Effect of high shear homogenization on rheology, microstructure and fractal dimension of acidinduced SPI gels. Journal of Food Engineering, 2014; 126: 48-55.

[26] Vicsek T. Fractal growth phenomena (Vol. 4). Singapore: World Scientific, 1989.

[27] Bi C H, Li D, Wang L J, Wang Y, Adhikari B. Characterization of nonlinear rheological behavior of SPIeFG dispersions using LAOS tests and FT rheology. Carbohydrate Polymers, 2013; 92(2): 1151-1158.

[28] Bi C H, Wang L, Li D, Huang Z G, Adhikari B, Chen X D. Non-linear rheological properties of soy protein isolate dispersions and acid-induced gels. International Journal of Food Engineering, 2017; 13(5). 